\title{
Elevated expression of ciRS-7 in peripheral blood mononuclear cells from rheumatoid arthritis patients
}

\author{
Xinyi Tang ${ }^{1}$, Jiemin Wang ${ }^{2}$, Xin Xia ${ }^{2}$, Jie Tian ${ }^{2 *}$, Ke Rui ${ }^{2}$, Huaxi X ${ }^{2}$ and Shengjun Wang ${ }^{1,2^{*}}$
}

\begin{abstract}
Background: Circular RNAs (circRNAs) represent a class of widespread and variety endogenous RNAs that may regulate gene expression. Thousands of mammalian circRNAs harbor miRNA response elements (MREs), suggesting a potential role as competitive endogenous RNAs (ceRNAs). Recent studies have demonstrated that ciRS-7 (circular CDR1 antisense), which acts as a powerful miR-7 sponge, contains more than 70 putative binding sites for miR-7 and may inhibit its target genes. The aim of this preliminary study was to investigate the expression of ciRS-7 in patients with rheumatoid arthritis (RA) as well as the correlation between ciRS-7 and the target genes of miR-7.

Methods: Eighteen patients with RA and 14 healthy controls were enrolled in the current study. The relative expression of ciRS-7, miR-7, miR-671 and mTOR in peripheral blood mononuclear cells (PBMCs) from these samples were detected by real-time PCR.

Results: We found that ciRS-7 was significantly increased in RA patients and could potentially differentiate the RA patients from healthy controls. Additionally, the expression of mTOR, one of the miR-7 target genes, had positive and negative relationships with ciRS-7 and miR-7 expression, respectively. Notably, the relative expression of miR671, which mediated the regulation of circular CDR1 antisense homeostasis, was significantly decreased in RA patients.
\end{abstract}

Conlusion: Downregulated miR-671 may influence the level of ciRS-7 in RA patients. Enhanced ciRS-7 could inhibit the function of miR-7 and further relieve the inhibitory effect of miR-7 on mTOR.

Keywords: ciRS-7, miR-7, mTOR, Rheumatoid arthritis

\section{Background}

Circular RNAs (circRNAs) are RNA molecules and important regulators of miRNA activity, which are formed by back-splice events and thus present as covalently closed continuous loops. MiRNAs are short, single stranded, non-coding RNAs involved in the post-transcriptional regulation of gene expression. ciRS-7 is one of the most widely studied circular RNAs [1, 2]. It has an antisense orientation with respect to the CDR1 gene, cerebellum degeneration-related antigen 1 (CDR34), which has been

\footnotetext{
*Correspondence: tij850913@163.com; sjwjs@ujs.edu.cn

${ }^{2}$ Institute of Laboratory Medicine, Jiangsu Key Laboratory for Laboratory

Medicine, Jiangsu University School of Medicine, Zhenjiang 212013, Jiangsu Province, China

'Department of Laboratory Medicine, The Affiliated People's Hospital, Jiangsu University, Zhenjiang 212002, China
}

implicated in autoimmune neurologic disorder since the late 1980s [3, 4]. Notably, ciRS-7 harbors more than 70 conventional miR-7 binding sites, acts as a designated miR-7 inhibitor/sponge, and efficiently reduces miR-7 activity, consistent with the competing eRNA (ceRNA) hypothesis [5]. However, in contrast to classical ceRNAs, ciRS-7 has no accessible termini and could be resistant to miRNA-mediated RNA destabilization [6]. This observation suggests that ciRS-7 might participate in various miR-7-dependent pathways in autoimmune diseases.

Rheumatoid arthritis (RA) is a chronic, inflammatory, autoimmune disorder characterized by synovial inflammation and adjacent cartilage and bone destruction that may result in severe lifelong disability $[7,8]$ ENREF_7. $\mathrm{RA}$ is driven by dysregulated innate and adaptive immune responses that provide an increasingly rich 
therapeutic resource. PI3K/Akt/mTOR is one of the most important intracellular signaling pathways in mammalian cells $[9,10]$. The role of the PI3K/AKT/mTOR pathway in promoting aggressive immune cell and synoviocyte proliferation and altered innate immunity in inflammatory arthritis was recently reviewed. [11-16] Importantly, a correlation was found between activated mTOR signaling and the number of osteoclasts in RA patients [17]. In addition, one miR-7 target site was found in the mTOR 3'UTR, and miR-7 was reported to efficiently regulate the PI3K/Akt pathway by targeting PIK3CD, mTOR and p70S6K $[18,19]$.

In this preliminary study, we aimed to detect the variation of ciRS-7 in RA patients and analyze the correlation between mTOR and ciRS-7 in peripheral blood mononuclear cells (PBMCs). Using this approach, we hoped to identify the potential diagnostic value of ciRS-7 in RA patients.

\section{Material and method}

\section{Patients and healthy controls}

A total of 18 patients with RA were enrolled in the study. The major clinical data of these patients are shown in Table 1. All patients met the American College of Rheumatology (ACR) 1987 and The European League against Rheumatism (EULAR) 2009 revised criteria for the classification of RA and without any treatment. All blood samples were collected under fasting conditions in the morning. The concentrations of anti-CCP antibody in the serum from RA patients were determined by ELISA (ORMOND, Germany) using an ELISA reader (Bio-Rad). Fourteen healthy control subjects were free of chronic pain, cardiovascular complaints, or other chronic inflammatory diseases. PBMCs were prepared from all patients and healthy controls for subsequent measurement.

All samples were collected in accordance with the regulations and approval of the Affiliated People's Hospital of Jiangsu University.

\section{RNA extraction and qRT-PCR}

PBMCs were isolated by density-gradient centrifugation over Ficoll-Hypaque solution [20]. TRIzol reagent (Invitrogen, Carlsbad, CA) was added to isolate PBMCs. Total RNA isolation was performed according to the

Table 1 Clinical features of RA patients included in this study

\begin{tabular}{llc}
\hline & RA & Range \\
\hline $\mathrm{n}$ & 18 & \\
Gender(F/M) & $12 / 6$ & $31-84$ \\
Age & $52.56 \pm 17.32$ & $1444-111,563$ \\
anti-CCP(IU/ml) & $26,840 \pm 34,351$ &
\end{tabular}

manufacturer's instruction. Reverse transcription (Toyobo, Osaka, Japan) was performed according to the manufacturer's instructions for synthesizing cDNA [21]. The primers in the reverse transcription kit (Toyobo, Osaka, Japan) consisted of oligo-dTN and random primers. The cDNA obtained by priming with random primers was used for ciRS-7 detection, and the cDNA obtained by priming with oligo-dTN was used for mTOR mRNA detection. The miRNA qRT-PCR Primer Set (RiboBio, Guangzhou, China) and M-MLV Reverse Transcriptase (TaKaRa, Dalian, China) were used for miR-7 and miR-671 reverse transcription.

qRT-PCR was performed in duplicate using the Bio-Rad SYBR Green Super mix (Bio-Rad, Hercules, CA). Primer sequences were as follows: mTOR, sense, 5'-CGCTGTCATCCCTTTATCG-3'; antisense, 5'-ATG CTCAAACACCTCCACC-3', ciRS-7, sense, 5'-CCTG GGCTCCTCGCCTGACC-3'; antisense, 5' - TCTCTC TGCCCTCAGCCTTGCC-3', GAPDH, sense, 5'-CAA GTC CCG CCG CTC CAT TAC CAA-3'; anti-sense, 5'-CCA CAG CCG TCC CAG TCA CAG TGG-3'. The expression levels of ciRS-7 and mTOR mRNA were normalized to the endogenous expression of GAPDH. The expression levels of miR-7 and miR-671 were normalized to the expression of U6. Data were analyzed by Bio-Rad CFX Manager software.

\section{Statistical analyses}

Student's unpaired or paired $t$ test was performed to determine whether there was a statistically significant difference between the RA group and healthy group. Correlations between variables were determined by Spearman's correlation coefficient. Receiver-operator characteristic (ROC) curve analysis was used to assess the diagnostic value, including AUC, sensitivity, and specificity. $P<0.05$ was considered statistically significant. All statistical analyses were performed with GraphPad Prism5 software (GraphPad Software, Inc., San Diego, CA).

\section{Results}

\section{Increased expression of ciRS-7 in patients with RA}

To study ciRS-7 expression in RA patients, peripheral blood was obtained from RA patients. As shown in Fig. 1a, the relative expression of ciRS-7 was significantly higher in patients than healthy controls. Increased anti-CCP antibody is a generally accepted objective clinical manifestation of RA patients. Numerous clinical studies have shown that anti-CCP antibody is an index of RA prognosis that is extremely specific for RA diagnosis. We analyzed the correlation of ciRS-7 expression with anti-CCP antibody concentration in RA patients and found a positive correlation between relative expression of ciRS-7 and plasma levels of anti-CCP antibody in RA patients (Fig. 1b). 

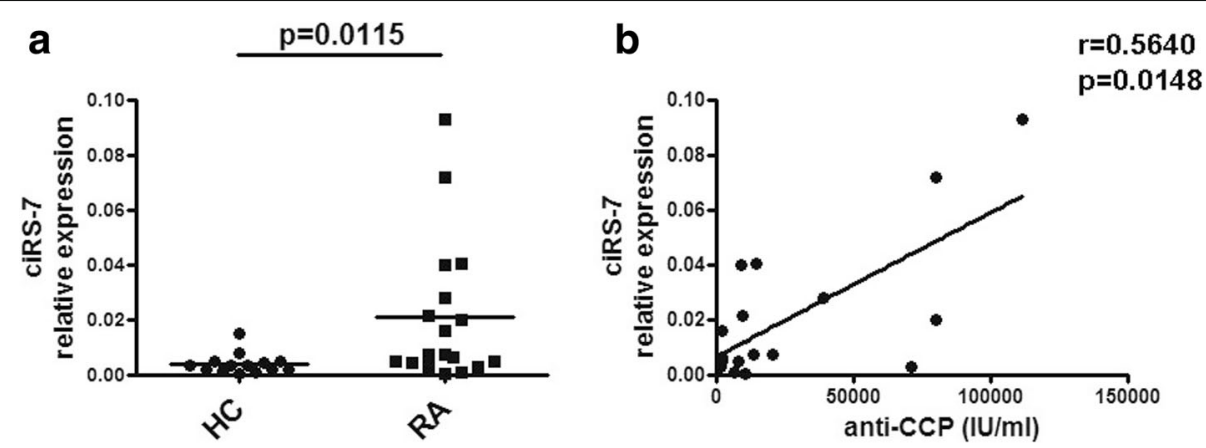

Fig. 1 Increased expression of ciRS-7 in patients with RA. a The relative expression of ciRS-7 in PBMCs from RA patients and controls was detected by QRT-PCR. $\mathbf{b}$ The correlation between the relative expression of ciRS-7 in PBMCs and concentration of anti-CCP in the serum from RA patients

The comparison of miR-7 expression between RA patients and control individuals

Increased ciRS-7 resulted in reduced miR-7 activity and increased levels of miR-7-targeted transcripts. The expression of ciRS-7 was increased in RA patients as described above, and we decided to examine the expression of ciRS-7. As shown in Fig. 2a, the relative expression of miR-7 significantly decreased in RA patients. miR-7a was reported to target the 3'-UTR of several components of the mTOR molecule and may modulate their expression and function. In this study, the relative expression of mTOR mRNA was detected in PBMCs from RA patients. The mTOR expression is higher in the PBMCs from RA patients compared with healthy controls (Fig. 2b). Notably, we found that expression of
mTOR in PBMCs from RA patients and healthy controls had a significant positive correlation with ciRS-7 expression and a negative correlation with miR-7 expression (Fig. 2c and d). Based on these results, we hypothesized that increased expression of ciRS-7 in RA peripheral blood may act as a sponge that could absorb a certain amount of miR-7 and further upregulate mTOR mRNA expression.

\section{Downregulation of miR-671 in RA patients}

CDR1 was identified as a putative target gene of miR-671. The target site is highly complementary to mature miR-671 and is situated in the antisense direction proximal to the CDR1 transcription start site [22]. As determined by northern blot analysis, ectopically expressed

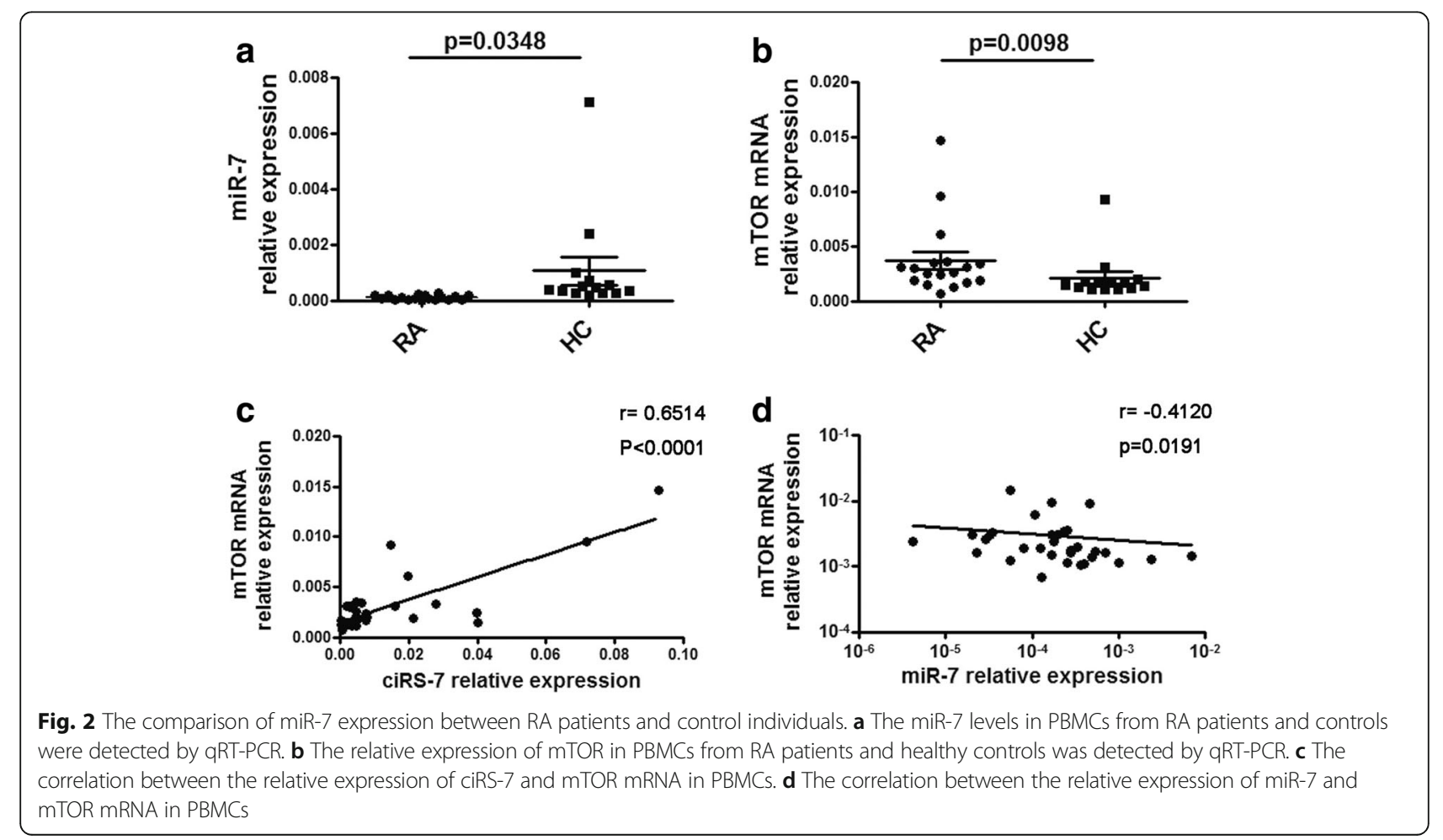


CDR1 antisense transcripts were almost completely eliminated by miR-671. Based on these results, the relative expression of miR-671 was detected in PBMCs from RA patients to explore the mechanisms of ciRS-7 enhancement. As shown in Fig. 3, the relative expression of miR-671 was significantly reduced in patients with RA.

\section{Evaluation of the diagnostic value of ciRS-7 for RA}

To assess the efficiency of ciRS-7 as a diagnostic marker for RA detection, we performed ROC curve analysis on ciRS-7. Figure 4 shows that the ROC curves of ciRS-7 could distinguish RA patients from healthy controls. For this, the sensitivity was $77.8 \%$ and the specificity was $78.6 \%$ with an AUC of 0.766 .

These data indicated that the ciRS-7, which showed the ability to differentiate RA patients from healthy controls, could act as a suitable biomarker for RA diagnosis.

\section{Discussion}

Several studies have elucidated the relationship between circRNAs and cancers [23, 24]. Cir-ITCH enhanced the expression of ITCH and further inhibited the Wnt/ $\beta$-catenin pathway. Interference of cir-ITCH may promote development of esophageal squamous cell carcinoma (ESCC) and colorectal cancer (CRC) through defective Wnt signaling $[25,26]$. Because of their stability, circRNAs are potential biomarkers for diagnosis of diseases. Thus far, no studies have examined the relationship between circRNAs and autoimmune diseases. Several researchers have shown that various microRNAs are involved RA [27-29]. Because these molecules can function as ceRNAs, alteration of circRNA expression may affect the function of related miRNAs and then alter the target genes of this miRNA. We hypothesized that changes in

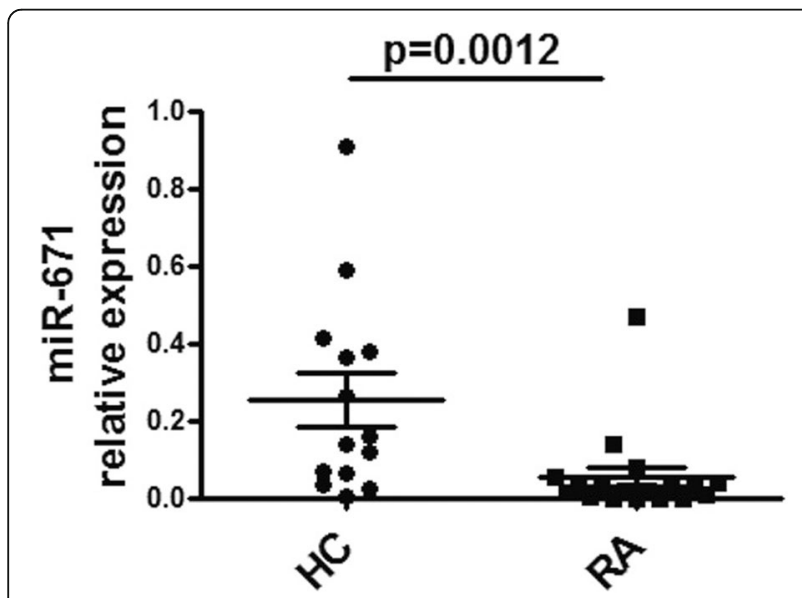

Fig. 3 Downregulation of miR-671 in RA patients. The expression of miR-671 in PBMCs from RA patients and healthy controls was detected by qRT-PCR

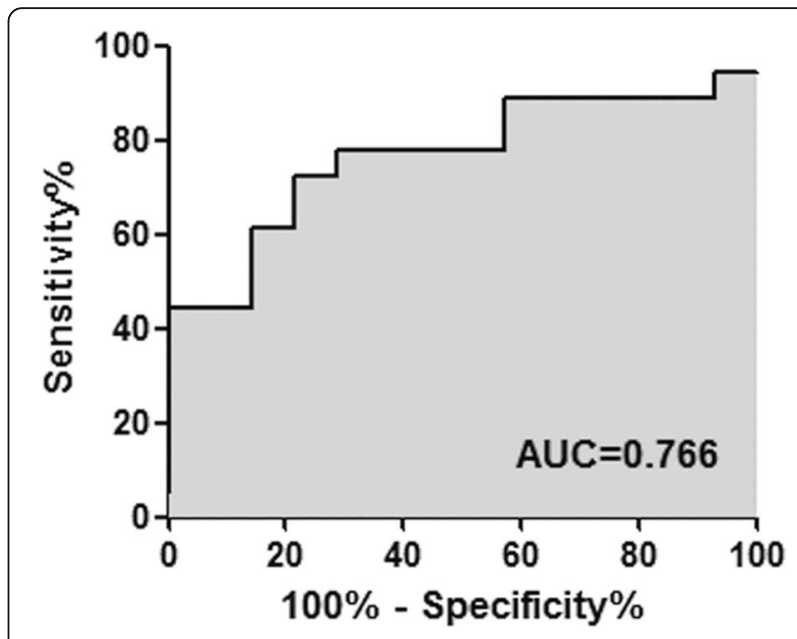

Fig. 4 ROC curve analysis using ciRS-7 to distinguish RA patients. ROC curve analysis was performed to show the AUC of ciRS-7

circRNAs may be involved the progression of RA through modification of miRNAs.

Interestingly, the results showed relative expression of ciRS-7 was significantly higher in peripheral blood from RA patients. Thus, we investigated the factors that caused this increase in ciRS-7 in patients with RA. A relevant article reported that inhibiting miR-671 by anti-mir-671 could activate CDR1 in HEK293 cells. Additionally, the researchers confirmed that CDR1NAS is cleaved and degraded in a miR-671-dependent manner [22]. Their data demonstrated that sequence-specific miR-671 mediated the regulation of circular CDR1 antisense homeostasis.

On the basis of these results, we detected the relative expression of miR-671 in the PBMCs from RA patients and compared them with healthy controls. Interestingly, the relative expression of miR-671 was significantly decreased in RA patients. Combined with the results of previous articles, we hypothesized that insufficient miR-671 in RA patients may be one factor that causes the high expression of ciRS-7.

To better elucidate the potential impact of enhanced ciRS-7 levels in the peripheral blood of patients with $\mathrm{RA}$, the expression of miR-7, which could be intensely absorbed by ciRS-7, was also detected. As expected, the relative expression of miR-7 in PBMCs from RA patients was significantly decreased compared with healthy controls. These results suggest that the miR-7 decrease was caused by elevated ciRS-7. However,no studies have shown that miR-7 that was absorbed by ciRS-7 could not be detected. Thus, determining the influence of ciRS-7 on the function of miR-7 and the expression of its target genes is more acceptable. The abnormal proliferation of synovial fibroblasts in RA patients was reported to be $\mathrm{PI} 3 \mathrm{~K} / \mathrm{mTOR}$ signaling-dependent. We 
investigated whether aberrant mTOR, one of the target genes of miR-7, was observed. Therefore, the relative expression of mTOR was detected. Consistent with our expectations, mTOR expression significantly increased in RA patients. Moreover, it had positive and negative relationship with ciRS-7 and miR-7 expression, respectively, indicating that ciRS-7, by indirectly influencing expression of mTOR in peripheral blood, may be involved in RA. However, the detailed mechanism remains unclear, and whether ciRS-7 destroys the synovial balance or is passively affected by other potential risk factors requires further investigation. Moreover, the direct evidence on the impact of ciRS-7 on the function of miR-7 is still unclear. We hypothesized that ciRS-7 may inhibit the function of miR-7. In addition, we assessed the efficiency of ciRS-7 as a diagnostic marker for RA detection, and the results indicated that the ciRS-7 level could potentially differentiate the RA patients from controls.

This study only reveals an interesting phenomenon and provides clues for further research on the diagnostic value of circular RNA in RA. However, there are still limitations of this study. In addition to comparing the relative expression of ciRS-7 in PBMCs from RA patients and healthy controls, the ciRS-7 expression should be compared in RA patients with or without treatment to study the value of ciRS-7 in disease treatment evaluation. Generally, downregulated miR-671 may influence the level of ciRS-7 in RA patients, and enhanced ciRS-7 likely inhibits the function of miR-7 and further relieved the inhibitory effect of miR-7 on mTOR.

\section{Conclusions}

To summarize, the level of ciRS-7 in RA patients may be influenced by lower expression of miR-671. Thereafter, enhanced ciRS-7 may inhibit the function of miR-7, and then further reduce the inhibitory effect of miR-7 on mTOR.

\section{Abbreviations \\ ceRNAs: Competitive endogenous RNAs; circRNAs: Circular RNAs; mTOR: Mammalian target of rapamycin; PBMCs: Peripheral blood mononuclear cells; qRT-PCR: Quantitative real time polymerase chain reaction; RA: Rheumatoid arthritis}

\section{Acknowledgements}

Not applicable.

\section{Funding}

This work was supported by the National Natural Science Foundation of China (Grant Nos. 31470881, 31711530025, 81701616), the Summit of the Six Top Talents Program of Jiangsu Province (Grant No. 2015-WSN-116), the Science and Technology Support Program (Social Development) of Zhenjiang (Grant Nos.SH2016043, SH2015045, SH2017008), the Jiangsu University Science Foundation (Grant Nos. 15JDG070).

\section{Availability of data and materials}

All data generated or analyzed during this study are included in the published article. The datasets used and/or analyzed in this study are available on request from the corresponding author.

\section{Authors' contributions}

XT designed the study, performed the experiment, participated in data statistics, and drafted the manuscript. JW performed the experiments. XX and KR collected PBMCs from RA patients. HX revised the manuscript for important intellectual content. SW and JT designed the study and revised the manuscript. All authors read and approved the final manuscript.

\section{Ethics approval and consent to participate}

This study was approved by the ethics committee of the Affiliated People's Hospital of Jiangsu University. Patients and healthy controls gave written informed consent prior to collection of their blood and tissue specimens.

\section{Consent for publication}

Not applicable.

\section{Competing interests}

The authors declare that they have no competing interests.

\section{Publisher's Note}

Springer Nature remains neutral with regard to jurisdictional claims in published maps and institutional affiliations.

Received: 27 August 2018 Accepted: 14 January 2019

Published online: 02 February 2019

\section{References}

1. Memczak S, Jens M, Elefsinioti A, Torti F, Krueger J, Rybak A, et al. Circular RNAs are a large class of animal RNAs with regulatory potency. Nature. 2013;495:333-8.

2. Hansen $T B$, Jensen $T I$, Clausen BH, Bramsen JB, Finsen B, Damgaard CK, et al. Natural RNA circles function as efficient microRNA sponges. Nature. 2013; 495:384-8.

3. Dropcho EJ, Chen YT, Posner JB, Old LJ. Cloning of a brain protein identified by autoantibodies from a patient with paraneoplastic cerebellar degeneration. Proc Natl Acad Sci U S A. 1987;84:4552-6.

4. Chen YT, Rettig WJ, Yenamandra AK, Kozak CA, Chaganti RS, Posner JB, et al. Cerebellar degeneration-related antigen: a highly conserved neuroectodermal marker mapped to chromosomes $X$ in human and mouse. Proc Natl Acad Sci U S A. 1990;87:3077-81.

5. Salmena L, Poliseno L, Tay Y, Kats L, Pandolfi PP. A ceRNA hypothesis: the Rosetta stone of a hidden RNA language? Cell. 2011;146:353-8.

6. Liu Y, Cui H, Wang W, Li L, Wang Z, Yang S, et al. Construction of circular miRNA sponges targeting miR-21 or miR-221 and demonstration of their excellent anticancer effects on malignant melanoma cells. Int J Biochem Cell Biol. 2013;45:2643-50.

7. Wang Y, Tian J, Wang S. The potential therapeutic role of myeloid-derived suppressor cells in autoimmune arthritis. Semin Arthritis Rheum. 2016;45: 490-5.

8. Wang S, Shi Y, Yang M, Ma J, Tian J, Chen J, et al. Glucocorticoid-induced tumor necrosis factor receptor family-related protein exacerbates collageninduced arthritis by enhancing the expansion of Th17 cells. Am J Pathol. 2012;180:1059-67.

9. Wang XW, Zhang YJ. Targeting mTOR network in colorectal cancer therapy. World J Gastroenterol. 2014;20:4178-88.

10. Willems L, Tamburini J, Chapuis N, Lacombe C, Mayeux P, Bouscary D. PI3K and mTOR signaling pathways in cancer: new data on targeted therapies. Curr Oncol Rep. 2012;14:129-38.

11. Banham-Hall E, Clatworthy MR, Okkenhaug K. The therapeutic potential for PI3K inhibitors in autoimmune rheumatic diseases. Open Rheumatol J. 2012; 6:245-58.

12. Beer-Hammer $S$, Zebedin $E$, von Holleben M, Alferink J, Reis B, Dresing $P$, et al. The catalytic PI3K isoforms p110gamma and p110delta contribute to $B$ cell development and maintenance, transformation, and proliferation. J Leukoc Biol. 2010;87:1083-95.

13. Choy E. Understanding the dynamics: pathways involved in the pathogenesis of rheumatoid arthritis. Rheumatology (Oxford). 2012;51(Suppl 5): $: 33-11$.

14. Malemud CJ. Intracellular signaling pathways in rheumatoid arthritis. J Clin Cell Immunol. 2013;4:160.

15. Nagai S, Kurebayashi Y, Koyasu S. Role of PI3K/Akt and mTOR complexes in Th17 cell differentiation. Ann N Y Acad Sci. 2013;1280:30-4. 
16. Ramadani F, Bolland DJ, Garcon F, Emery JL, Vanhaesebroeck B, Corcoran $A E$, et al. The PI3K isoforms p110alpha and p110delta are essential for pre-B cell receptor signaling and B cell development. Sci Signal. 2010;3:ra60.

17. Wu T, Mohan C. The AKT axis as a therapeutic target in autoimmune diseases. Endocr Metab Immune Disord Drug Targets. 2009;9:145-50.

18. Wang Y, Liu J, Liu C, Naji A, Stoffers DA. MicroRNA-7 regulates the mTOR pathway and proliferation in adult pancreatic beta-cells. Diabetes. 2013;62: 887-95.

19. Fang Y, Xue JL, Shen Q, Chen J, Tian L. MicroRNA-7 inhibits tumor growth and metastasis by targeting the phosphoinositide 3-kinase/Akt pathway in hepatocellular carcinoma. Hepatology. 2012;55:1852-62.

20. Tian X, Ma J, Wang T, Tian J, Zhang Y, Mao L, et al. Long non-coding RNA HOXA transcript antisense RNA myeloid-specific 1-HOXA1 Axis downregulates the immunosuppressive activity of myeloid-derived suppressor cells in lung Cancer. Front Immunol. 2018:9:473.

21. Tian J, Rui K, Hong Y, Wang X, Xiao F, Lin X, et al. Increased GITRL impairs the function of myeloid-derived suppressor cells and exacerbates primary Sjögren's syndrome. J Immunol; 2019. https://doi.org/10.4049/jimmunol. 1801051.

22. Hansen TB, Wiklund ED, Bramsen JB, Villadsen SB, Statham AL, Clark SJ, et al. miRNA-dependent gene silencing involving Ago2-mediated cleavage of a circular antisense RNA. EMBO J. 2011:30:4414-22.

23. Zhong Z, Huang M, Lv M, He Y, Duan C, Zhang L, et al. Circular RNA MYLK as a competing endogenous RNA promotes bladder cancer progression through modulating VEGFANEGFR2 signaling pathway. Cancer Lett. 2017; 403:305-17.

24. Chen J, Li Y, Zheng Q, Bao C, He J, Chen B, et al. Circular RNA profile identifies circPVT1 as a proliferative factor and prognostic marker in gastric cancer. Cancer Lett. 2017;388:208-19.

25. Li F, Zhang L, Li W, Deng J, Zheng J, An M, et al. Circular RNA ITCH has inhibitory effect on ESCC by suppressing the Wnt/beta-catenin pathway. Oncotarget. 2015;6:6001-13.

26. Huang G, Zhu H, Shi Y, Wu W, Cai H, Chen X. Cir-ITCH plays an inhibitory role in colorectal cancer by regulating the Wnt/beta-catenin pathway. PLoS One. 2015:10:e0131225.

27. Chen JQ, Papp G, Szodoray P, Zeher M. The role of microRNAs in the pathogenesis of autoimmune diseases. Autoimmun Rev. 2016:15:1171-80.

28. Moran-Moguel MC, Petarra-Del Rio S, Mayorquin-Galvan EE, Zavala-Cerna MG. Rheumatoid arthritis and miRNAs: a critical review through a functional view. J Immunol Res. 2018;2018:2474529.

29. Hong BK, You S, Yoo SA, Park D, Hwang D, Cho CS, et al. MicroRNA-143 and -145 modulate the phenotype of synovial fibroblasts in rheumatoid arthritis. Exp Mol Med. 2017;49:e363.

Ready to submit your research? Choose BMC and benefit from:

- fast, convenient online submission

- thorough peer review by experienced researchers in your field

- rapid publication on acceptance

- support for research data, including large and complex data types

- gold Open Access which fosters wider collaboration and increased citations

- maximum visibility for your research: over $100 \mathrm{M}$ website views per year

At $\mathrm{BMC}$, research is always in progress.

Learn more biomedcentral.com/submissions 